\title{
Information Technology and Telecommunication Infrastructure: Network Applications for Hong Kong Business and Service
}

\author{
A. A. Shabayek, Kin-man Wan \\ University of Hong Kong, Hong Kong, China \\ Em:aaeshaba@hkucc.hku.hk,Em:kmwan@graduate.hku.hk
}

Keywords Broadband Network, Information Technology, Multimedia Infrastructure

\begin{abstract}
Hong Kong is an unique city in being very well served by robust communications networks; in particular the telephone and cable television networks can provide high capacity optical fibre links to virtually all premises at low cost. Hong Kong needs to make the best use of the ever-advancing information and communication network to improve products as well as services for public and private sectors. With the network in place, the missing link is integrating different elements in the system. Integrated multimedia system is an example of a niche market that is yet untapped. This paper focuses at the elements that encompass the integration of multimedia infrastructure for Hong Kong industry and service and also outlines applications demonstrating the linkage of different networks.
\end{abstract}

\section{INTRODUCTION}

Information technology have been dominated by the advanced industrialised countries, and large companies, but recent technological advances are opening up large market sectors and permitting new players to participate [1]. Our very up-to-date communication network, the compact size of the territory and its position as an important commercial and financial centre combine to give Hong Kong the unique opportunity to develop and implement new communication services to become a leading digital city in the world. This opportunity is presented as an example to illustrate our thesis that, even with limited R\&D investment, very significant new business prospects could be generated. In the information and telecommunication sector, many businesses are already flourishing in Hong 
Kong. They have a synergistic effect on the potential of developing new businesses. Each existing business already has its invested capital and growth potential and in some cases, also significant R\&D know-how. The key issue is to evaluate, against the current background, the additional investment needed in $R \& D$ in order to reach various selected business opportunities. Then the specific capital investment requirements for a chosen new business can be determined.

In early 1990s the telecommunication network was primarily telephony based. In recent years, technological advances have extended the information-carrying capabilities of the network first through the introduction of digital techniques and then through all-electronic switching. Many new switched data services have appeared [2].

With the graduate widespread installation of optical fibre system in the trunk and distribution areas of the public and private networks, the stage is set for the networks to handle a vast range of new services. These services may differ significantly in nature from the switched telephony service. The network would be capable of handling broadband signals such as video, thereby introducing a degree of conflict between TV distribution and telephony [3]; the conflict could also be viewed positively as opportunity to merge certain services.

\section{NETWORKING CONSTITUENCIES}

Mainly equipment makers, network operators, and Service vendors are the key players supporting the networking system. Traditionally, equipment makers in the telecommunication industry work closely with the carrier network operators and in anticipation of the service demands of the customers; they design and manufacture customer premises, systems and network equipment [4]. Vast investments are made in technology R\&D with the aim of extending the capabilities of the network for service delivery at better quality and lower cost. This healthy and productive marriage between the equipment makers and the network operators has benefited the customers well.

Common carriers operate in a regulated environment; they are obliged to connect all customers, however remote, to the telecommunications network. In the current environment, the customers may connect approved equipment such as telephones, computers and fax machines to the network. Carrier operators charge the calls by time, distance and bandwidth at a rate approved by the regulatory commission. The approval rate on justifiable capital and operation costs and acceptable profit margin. The common carrier operators strive to satisfy all customer demands at a low charge per call, with little or 
no waiting time, and with many convenient call features. This is achieved by working with the equipment suppliers to improve call features and to reduce cost per call through technological improvement [5].

\section{TELECOMMUNICATION-RELATED BUSINESS IN HONG KONG}

The First Pacific group is entering the telecommunication business along several avenues. Pacific Link Communication is the newest of the three cellular phone operators. First Pacific Mobile Communication offers trunked radio service and has obtained a license to provide paging service. In partnership with three other companies, it is bidding for a license to offer CT2 service in Hong Kong.

Hutchinson Telecommunications is expanding forcefully and rapidly in several fields of telecommunications. It owns $50 \%$ of the communication satellite AsiaSat 1 and will be broadcasting TV programs to a large part of Asia. Hutchinson Telephone operates two cellular networks with the dominant market share among the three cellular operators. Hutchinson Paging offers paging service to $50 \%$ of the market and is bidding with partners for a CT2 license. Hutchinson iNET offers a value-added electronic mail service and Hutchinson Mobile Data offers financial information through a radio broadcast to subscribers.

In 1990, Hutchinson offered the first trunked radio communication (TRC on $800 \mathrm{MHz}$ frequency to provide more efficient radio communication to taxis and commercial fleets. Hutchinson is also expanding overseas. For example, it has established a foothold in the UK by buying the paging company Millicom Information Services and by seeking a controlling share in BYPS, a CT2 service provider [6].

There are four cellular networks in service in Hong Kong: the UNITACS system of HKT CSL; the ETACS system of Pacific Link; and the AMPS and TACS systems of Hutchinson. As of year-end 1999, there were 670,000 subscribers, or more than $10 \%$ of Hong Kong's population, yielding the highest penetration rate of hand-held cellular sets in the world. With an annual growth rate of $47 \%$, spurred by up to $50 \%$ annual price drops on handsets, it is expected that the four systems will be saturated by year-end of 2000. To provide a long-term solution, The Hong Kong Government is providing the digital cellular system via the American USDC and the European GSM standard. 


\section{IT APPLICATIONS VIA BROADBAND TELECOMMUNICATIONS NETWORKS}

Information Technology (IT) emerges to make Hong Kong becoming one of the leading digital cities in the world. The Technology applies in many different areas, which support the enhancement of the industry as well as services and maintain Hong Kong at the competitive edge in the information arena. In order to enhance work's accuracy and efficiency on preparing and retrieving client records, centralised databases system and client/server computing environment enable manufacturers, hospitals, banks, and government department in Hong Kong to check and update their records efficiently at no time.

\subsection{Medical Service}

Due to the development of advanced information technology in medical services, the Hospital Authority in Hong Kong is continuously up-dating the medical service by installing sophisticated information systems and devices through the medical broadband network, e.g. medical imaging and scanning machines. The medical network in Hong Kong has a number of Computerised Tomography scanners (CT) and Magnetic Resonance Imager (MRI). Other medical imaging tools include ultrasound and Nuclear Medicine (NM).

Given Hong Kong 's scarcity of CT's and MRI's, its need to maximise the utility of limited medical expertise, and the needs of the hospitals to upgrade and/or computerise their management and technical capabilities, a medical broadband network would be useful. The main purpose of such a medical broadband network are to share resources, provide timely access to data and pictures, and allow easier access of expertise to support medical decision. The network allows major hospitals to pool their radiology/pathology facilities and share expensive and scarce medical expertise. Broadband communication can enhance the utilisation of such equipment and such specialists' time and lower the pre-patient cost by spreading the overheads a lager user community.

Due to the large number of films and operational necessity to physically transfer these films among departments and doctors, an estimated $30 \%$ of these records are lost. A computerised picture archive and communication system (PACS) would process and store digital records of X-ray, ultrasound, CT, MRI and NM images in optical disk storage [7]. Figure 1 depicts the 
general concept of a broadband bus that links hospitals to centres of shared facilities.

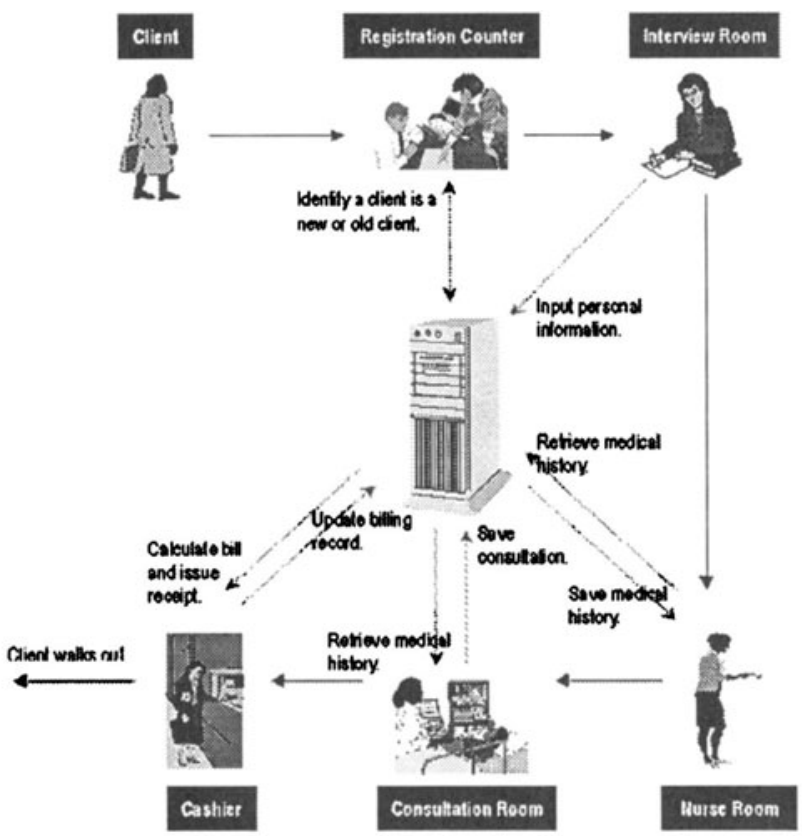

Figure 1 Medical Broadband Network Linking Hospitals, Clinics and Doctor Offices to Centres Shared Facilities, e.g. Radiology \& Pathology Labs.

\subsection{Transportation Infrastructure Network Via IT \& Smart Card System}

Hong Kong has very reliable transportation infrastructure via development of information technology into their system. The Transport Department is now expanding the transport system with the introduction of an up-to-date intelligent support system to reduce traffic congestion and travelling time. All of these facilities can bring a great convenience to the citizens of Hong Kong. Traditionally, the contact magnetic card is the only ticketing system of public transportation. After the introduction of the automated fare collection system, the process became much easier and user friendly. By using the Octopus transit card and the imaging processing technology, the passengers can travel conveniently on different modes of public transportation systems. The system design is based on the integration of ticketing network on different modes of public transportation. As a result, the operating and maintenance costs can be reduced.

The octopus transit card has an embedded microprocessor that stores, updates, and transfers information, in real time, to the transient networks. 
For the passengers' convenience, information transmission to the reader of the transportation module occurs instantaneously even with no direct contact with the smart-card, e.g. scanning the passenger's wallet which contains the octopus card is enough for communication. Once communication between imbedded antenna (smart card) and external antenna (reader) started, the card's chip becomes energised due to the magnetic induction and the magnetic waves and radio signals transmit the data.

Each time the smart card is used, its credit status is updated and transaction data is processed immediately to computerized station of the main system [8]. The system then sends the data to credit analyzers, addvalue machines, auditor gates and integrated processors. There is a central clearinghouse that connects all the computers using wide area network (WAN). All transaction data is transmitted to the clearinghouse for checking, processing and distributing. If passenger wants to add value to her/his smart card, she/he can use the add-value machine, to reload extra credit into the octopus's microprocessor. At the same time, the updated data will be transmitted to the whole system, i.e. credit analyzers, processors, etc. A flow chart summarizes this process is shown below in Figure 2.

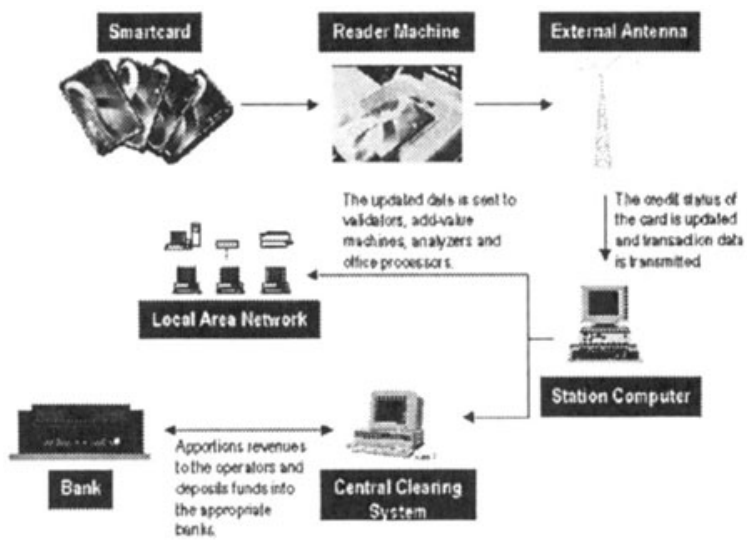

Figure 2 Flowchart for Octopus Transit Network.

\subsection{Intelligent Traffic System}

Hong Kong, as a whole, comprises group of islands that are interconnected by bridges and underwater tunnels. In order to optimise traffic on highways more effectively, Hong Kong is developing more intelligent transport system such as traffic control system, automatic toll collection (auto-pass) system, and satellite speeding control system. The traffic control system acts as a safety valves in the traffic networks; it is 
equipped with infrared sensors that measure traffic congestion and control traffic lights accordingly. While the main function of the automatic toll or auto-pass system is to effectively and automatically identify each passing vehicle through toll roads, bridges or tunnels in order to process the charge electronically. Electronic road pricing system is a new technology that offers an optimised way to use roads and highways. The system uses microwave signals via local area network (LAN) as well as wide area network (WAN) and microwave towers to detect the vehicles' identification number (ID) that cross the pricing location for automatic billing. Usually, the signal block sends a signal to the passing vehicle, which is equipped with a smart card placed at the vehicle's front. Once the signal scanner scans the smart card, the signal reflects back the information to the system with the required fee debited from the smart card's account. Similarly, the satellite speeding system uses a radar detector for speed measurements and also identifies any over speeding vehicle; consequently the system transmits the information electronically via WAN and microwave towers to the traffic bureau's main computer for processing [9].

\subsection{IT for The Airport Flight System}

The Hong Kong new International Airport, at Chek Lap Kok, implemented the most sophisticated IT in its ground and flight operation systems. The operation system includes check-in ticketing system, gate arrangement system, flight information monitoring system, luggage checking and handling system, Cargo transportation system, etc.

The Hong Kong Civil Aviation Department applies different information technologies to control operations within the airport as well as air traffic in order to enhance flight safety. Advanced radar system and communication network can provide updated and accurate information to the pilots' cockpit and hence maintain orderly flows of air traffic. The radar flight system provides important information such as accurate aircraft position, road map for takeoff and landing, and the movement of vehicle on the runways. Such information is also transmitted to traffic controllers for flight routing arrangement. With this sophisticated system, pilots and controllers would be able to maintain a smooth and safe traffic even under low visibility conditions. The Hong Kong Civil Aviation Department is continuously improving the air traffic control by using satellite system for monitoring the flight routing and air traffic [10].

Radar and satellite systems enable the pilots and controllers to exchange information in real-time basis. This information includes weather condition, altitude, wind velocity, relative position of aircraft, etc. A geosynchronous 
satellite, moves with the earth at the same speed and direction, calculates the position of the aircraft and disseminates the information to the whole system as air traffic signals, e.g. giving map for another route in order to avoids collision with close-by aeroplanes. Figure 3 outlines the flow of information for flight monitoring system.

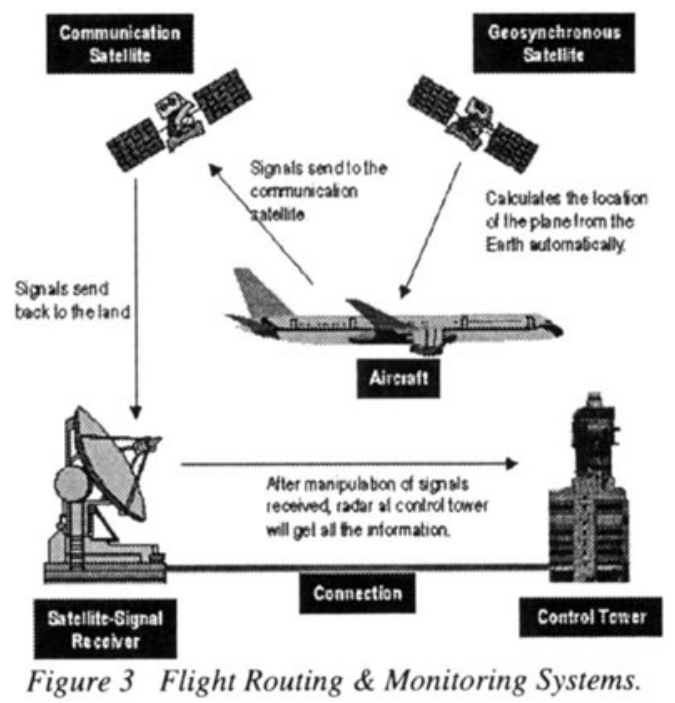

\section{CONCLUSION}

This paper identifies integrated multimedia information system for small as well as large business to be one niche market. Hong Kong s ideally placed to enter the market because the system is designed/application intensive and there is ready access to low-cost manufacturing and software development in China.

In real economic terms, the proposed development will create business opportunities for a core of networks and communication equipment manufacturers. The results of $R \& D$ will spread to related industry and downstream manufacturers. In the long term, a road map and well thought out plan would minimise the development risk and maximise the product evolution flexibility. For example, hardware development should make use of application-specific integrated circuit (ASIC) and software for ease of upgrade.

A graphic database service could be the next service for development. The distributed vector databases and graphic CAD tools would give rise to many unfamiliar operational issues, here in Hong Kong, and possibly a range of new customer premises equipment. This would be the basis for a 
range of service that could improve design and production efficiencies for various industries and would open up the path towards developing a host of multimedia services.

\section{REFERENCES}

[1] National Science and Technology Council. (1996), Technology in the National Interest, Office of Technology Policy, U.S. Department of Commerce, Washington, DC

[2] Industrialist, (Jan. 1999), Monthly Industry Magazine Published By The Federation of Hong Kong Industries, 37-39

[3] Telecommunication Internetworking (1999). Louis P. J, McGraw-Hill Publishing Group

[4] Chen Y.B., Li X., Orady E. (1996). Integrated Diagnosis Using Information-GainWeighted radial Basis Function Neural Networks, Computers and Industrial Engineering, 30(2), 243-255

[5] Sollenberg N. R. (1997). Business \& Technology Trends , Business \& Technology Information Quarterly, Hong Kong, 3(1), 16-20

[6] IEEE Spectrum, Sept. (1996)

[7] Hong Kong Health and Welfare Bureau, http://www.info.gov.hk/hwb/index.htm

[8] Hong Kong Transport Bureau, http://www.info.govhk/tb/

[9] Hong Kong Highway Department, http://www.hyd.gov.hkl

[10] Hong Kong Airport Authority, http://www.hkairport.com/ 